\title{
Incidência de infecção em pacientes com cateter peridural tunelizado
}

\author{
Infection incidence in patients with tunneled peridural catheter \\ Incidencia de infección en pacientes con cateter peridural tunelizado
}

\section{Maria Cecilia Iksilara}

Enfermeira responsável pelo Centro de Dor e Neurocirurgia Funcional do Hospital Nove de

Julho. Mestranda em Enfermagem pela Universidade Federal de São Paulo (UNIFESP).

Solange Diccini

Enfermeira. Professora Adjunto da Disciplina de Fundamentos de Enfermagem e Enfermagem médico-cirúrgica do Departamento de Enfermagem da Universidade Federal de São Paulo (UNIFESP).

\section{Dulce Aparecida Barbosa}

Enfermeira. Professora Adjunto da Disciplina de Fundamentos de Enfermagem e Enfermagem médico-cirúrgica do Departamento de Enfermagem da Universidade Federal de São Paulo (UNIFESP).

\section{RESUMO}

O cateter peridural tunelizado como via de administração de opiáceos vem sendo utilizado desde 0 início de 1980. Pacientes com dor crônica, que não obtêm alívio com medicamentos por outras vias, são muito beneficiados com infusão de opiáceo associado a um anestésico local por via peridural. Entretanto, ainda existem dúvidas sobre a eficácia do método, no manuseio e, conseqüentemente, quanto ao risco de infecção e outras complicações. Sendo a equipe de enfermagem fundamental para efetivar o tratamento para o alívio da dor, esse estudo propõe demonstrar como manter a técnica segura. Foram acompanhados 27 pacientes com dor crônica entre 2002 e 2004, que utilizaram o cateter peridural por 18 dias em média, implantados em nível torácico ou lombar. Não houve complicações como abcesso peridural, meningite ou hematoma peridural. A satisfação dos pacientes quanto a analgesia foi evidente.

Descritores: Analgesia; Analgesia epidural; Infecção.

\section{ABSTRACT}

The tunneled epidural catheter as an administration access for opiates has been used since the beginning of 1980. Patients with chronic pain, who does not get relief with medication through other accesses are benefited with infusion of opiate associated to a local anesthetic through epidural access. However, there are still doubts on the efficacy of the method in the handling and, consequently, over the risk for infection and other complications. As nursing is fundamental to make effective the relieve pain treatment, this study proposes to demonstrate how to keep safe the technique. Twenty-seven patients with chronic pain using epidural catheter for 18 days were followed between 2002 and 2004. Catheters were implanted in thoracic or lumbar level. No complications like epidural abscess, meningitis our epidural haematoma were observed. Patients' satisfaction related to analgesia was evident.

Descriptors: Analgesia; Analgesia, epidural; Infection.

\section{ABSTRACT}

El cateter peridural tunelizado, como via de administración de opióides, es utilizado desde el comienzo de 1980. Pacientes con dolor crónico, que no tienen alivio con medicamentos por otras vias, son muy beneficiados con la administración de opióides, asociados a un anestésico local por via peridural. Sin embargo, aún existen dudas sobre la eficacia del método, en el manejo y, como consecuencia, en el riesgo de infección y otras complicaciones. Siendo el equipo de enfermería fundamental para ejecutar el tratamiento para alivio del dolor, esto estudio se propone a presentar como mantener la técnica segura. Vinte y siete pacientes con dolor crónica, entre 2002 a 2004, que utilizarón el cateter peridural por 18 dias en media, fuerón seguidos. Los cateteres fuerón implantados al nivele toracico o lumbar. No se presentaran complicaciones como absceso peridural, meningitis o hematoma peridural. La satisfacción de los pacientes quanto analgesia fué evidente.

Descriptores: Analgesia; Analgesia epidural; Infección.

Iksilara MC, Diccini S, Barbosa DA. Incidência de infecção em pacientes com cateter peridural tunelizado. Rev Bras Enferm 2005 mar-abr; 58(2):152-5.

\section{INTRODUÇÃo}

A analgesia peridural foi utilizada pela primeira vez em 1885 por Corning, que teve a idéia de que os medicamentos injetados dentro do canal espinhal pudessem ser absorvidos pelos plexos de vasos sangüíneos da vizinhança e serem transportados para a substância da medula raquiana, fornecendo uma analgesia cirúrgica. Em 1901, Tuffier tentou a analgesia peridural pela via de acesso lombar, mas não obteve sucesso. Foram décadas de tentativas, mas a maioria dos anestesiologistas achava que era um método pouco seguro e até mesmo perigoso. Fidel Pagés, em 1921, renovou o interesse pelo acesso lombar, salientando a maior facilidade de acesso e ampla aplicabilidade dessa via em comparação a via caudal. Em 1949, Curbello, com a introdução 
da agulha de Tuohy e do cateter peridural de demora, promoveu um refinamento da técnica, tornando-se possível manter a analgesia contínua ou intermitente, estendendo-se por dias ou semanas, se necessário ${ }^{(1)}$.

Atualmente, a via peridural é segura e efetiva para administração de opióides, principalmente quando a administração de analgésicos por via oral ou venosa não proporcionam alívio satisfatório da dor. Além do rápido efeito com menor concentração de analgésicos, pode-se associar um anestésico local, que quando administrados no espaço peridural ligam-se aos receptores de morfina do sistema nervoso central (SNC) e do sistema nervoso periférico (SNP), proporcionando analgesia de longa duração sem bloquear as vias sensitivas, neurovegetativas simpática e $\operatorname{motora}^{(2,3)}$.

Bonica, o precursor dos modernos estudos sobre dor, valorizava muito o tratamento da dor crônica e, após muito estudo e por uma experiência pessoal, notou que o tratamento da dor aguda é igualmente importante ${ }^{(3)}$. A dor pós-operatória é o exemplo mais freqüente de dor aguda e até 0 século passado, era aceita pelo paciente com resignação. Hoje é considerada omissão da equipe médica ou até desinformação sobre farmacologia ${ }^{(4)}$.

Em cirurgias de grande porte, quando utilizada a anestesia peridural contínua, o mesmo cateter pode ser mantido no pós-operatório para infusão de solução analgésica, proporcionando uma analgesia eficaz. As vantagens evidentes de uma boa analgesia são a cooperação do paciente nos exercícios respiratórios e a deambulação precoce, diminuindo o risco de complicações como atelectasias, pneumonias, trombose venosa profunda, entre outras ${ }^{(5)}$.

A dor crônica é a segunda causa de procura pela assistência médica ${ }^{(6)}$. Geralmente está relacionada com lesão do sistema nervoso e, além de gerar incapacidade, interfere no estudo, trabalho, apetite, lazer e sono. $O$ tratamento depende de uma boa avaliação, analgésicos adequados, exercícios fisioterápicos e, muitas vezes, de acompanhamento psicológico. É comum o paciente necessitar de medicações em altas doses por via oral, o que não garante o alívio da dor. Nesses casos, há indicação do implante do cateter peridural por tempo indeterminado, utilizando a técnica de tunelização(7), que consiste em, após o implante do cateter no espaço peridural, fazer um trajeto lateralmente ao local da punção pelo subcutâneo, até atingir a região do hipocôndrio, onde é fixado.

No início da década de 80, o cateter tunelizado foi utilizado para os pacientes com dor crônica intensa, que necessitavam uso prolongado de morfina, principalmente nas neoplasias ${ }^{(8)}$. Atualmente, também é utilizado para dores causadas por úlceras e neuropatias diabéticas por insuficiência arterial periférica, fibromialgia, fraturas vertebrais, lombociatalgias, entre outras.

A tunelização diminui o risco de deslocamento, facilitando a movimentação do paciente e permitindo fazer a higiene pessoal normalmente. É possível fazer a troca do curativo, sempre que necessário, o que diminui o risco de infecção ${ }^{(7-9)}$, que é a maior complicação do uso dessa via. Caso não seja detectado a tempo, pode evoluir para um abcesso peridural(10-12), com incidência de $0,05 \%{ }^{(13)}$.

Além da infecção, outras complicações relacionadas ao cateter peridural tunelizado são a migração do cateter para 0 espaço subaracnoideo, saída acidental, dobra e quebra ${ }^{(14,15)}$.

As contra-indicações para o implante do cateter peridural são pacientes que fazem uso de anticoagulantes e antiinflamatórios, por causarem disfunção plaquetária com conseqüente risco de hematoma; anormalidades importantes na coluna vertebral que dificultem a passagem do cateter e a falta de profissionais de enfermagem treinados para freqüente avaliação desses pacientes ${ }^{(8)}$.

Na década de 1950, vários centros médicos norte-americanos foram criados no intuito de melhorar o tratamento da dor crônica. Entre 1960 e 1970, surgiram clínicas de dor na Europa e nos EUA e, dez anos depois, tornou-se uma obrigatoriedade a existência de grupos especializados para assistência ao paciente com dor nos hospitais ${ }^{(16,17)}$. Para isso, é necessário um profissional enfermeiro nos Centros de Dor, uma tendência nos hospitais atualmente.

No início de 1990, no Hospital Nove de Julho, um grupo de neurologistas e neurocirurgiões começou a tratar pacientes com dor crônica, mas foi em 2000 que foi oficilaizado o Centro de Dor na instituição, para o tratamento da dor aguda e crônica. Nesse mesmo ano, uma enfermeira foi designada para coordenar o Serviço, com equipe multiprofissional, entre eles, acupunturista, fisioterapeutas, psiquiatra e psicólogas. Hoje, uma área do hospital está disponível para assistência às pessoas portadoras de qualquer tipo de dor, com um número maior de profissionais, visando a melhora global dos pacientes.

Para um manejo eficaz da dor, o enfermeiro deve ter conhecimento sobre teoria e conceitos de dor, fisiologia dos trajetos de dor e da medula espinhal, métodos de avaliação e mensuração da dor, manejo farmacológico e não-farmacológico da dor e prevenção e manejo de complicações ${ }^{(4)}$.

Na nossa vivência, o alívio da dor com cateter peridural tunelizado é excelente e com poucos efeitos colaterais, tendo uma relação riscobenefício favorável e melhora da qualidade de vida, porém o receio de infecção e dúvidas no manuseio e na troca do curativo ainda é muito evidente por parte dos profissionais de saúde. A atuação do enfermeiro é fundamental, não só pelo aspecto da humanização ao aliviar a dor, como na educação e supervisão da equipe de enfermagem em relação a manutenção do cateter peridural, proporcionando um atendimento com qualidade.

\section{OBJETIVO}

O objetivo do trabalho foi avaliar a incidência de infecção no uso do cateter peridural tunelizado, utilizado para analgesia de pacientes com dor crônica.

\section{MÉTODO}

Estudo coorte prospectivo, foi realizado de novembro de 2002 a julho de 2004, no Hospital Nove de Julho, na cidade de São Paulo. Os critérios de inclusão compreenderam pacientes de ambos os sexos, de 15 a 90 anos, com cateter peridural tunelizado instalado, com tempo de permanência maior ou igual a 3 dias. Foram excluídos os pacientes que apresentavam alguma infecção confirmada em outro sítio, através de exames laboratoriais e pela Comissão de Controle de Infecção Hospitalar. O projeto de pesquisa foi submetido e aprovado pelo Comitê de Ética em Pesquisa do Hospital Nove de Julho e da Universidade Federal de São Paulo. Os pacientes incluídos nesse estudo assinaram o termo de consentimento livre e esclarecido. A indicação do implante do cateter peridural e sua retirada obedeceram a critérios médicos e, depois de instalado, foi acompanhado pela enfermeira responsável pelo Centro de Dor do hospital.

Na instituição do estudo, o cateter peridural utilizado para a técnica de tunelização foi da marca Arrow®, próprio para longa permanência, com infusão contínua de solução analgésica através de bomba infusora ou intermitente, em bolus com seringa. 0 cateter foi implantado pelo médico anestesista, no centro cirúrgico, com paramentação. Dependendo do estado geral do paciente, permanece internado ou tem alta hospitalar para acompanhamento ambulatorial, com um retorno semanal.

A técnica de passagem do cateter peridural consiste em punção lenta e cuidadosa com agulha Tuohy no espaço intervertebral, próximo a área que se deseja analgesia. Posiciona-se o paciente sentado ou em decúbito lateral com a cabeça fletida, realiza-se anti-sepsia da pele da região dorsal com polivinilpirrolidona-Iodo (PVPI) e anestesia local. 
Localizado o espaço peridural, implanta-se o cateter e retira-se a agulha. Lateralmente à punção, fazendo um túnel no subcutâneo com a própria agulha, o cateter é passado até sair na região do hipocôndrio. Na saída do cateter, são feitos pontos de mononylon 3,0 para melhor fixação, conecta-se 0 filtro antibacteriano e faz-se curativo com fita adesiva hipoalergênica à base de papel.

O instrumento de coleta de dados incluiu registros sóciodemográficos e informações como data da internação, diagnóstico da doença primária e secundária e uso de antibiótico, presença de cateter central, sonda vesical, dreno pleural ou outros. Dados referentes ao cateter peridural, como data do implante, nível de inserção, intercorrências no manuseio, presença de sinais flogísticos no local da inserção, sintomas de infecção sistêmica, tempo de permanência do cateter e motivo da retirada.

O curativo foi trocado pela enfermeira da unidade de internação ou do Centro de Dor, quando estivesse úmido ou solto e a solução analgésica também preparada e trocada pelas enfermeiras. Para a troca do curativo, foram utilizados soro fisiológico 0,9\% e PVPI tópico no local da inserção e sendo feito a cobertura com fita adesiva hipoalergênica à base de papel. Em seguida, o filtro antibacteriano era fixado no abdome também com a mesma fita adesiva.

Quando solicitada a retirada do cateter peridural, a ponta era enviada para o laboratório para cultura. 0 paciente era orientado quanto ao procedimento e posicionado sentado ou em decúbito lateral. O curativo era removido e feito a anti-sepsia da pele ao redor da inserção com PVPI tópico. Apenas $5 \mathrm{~cm}$ da ponta inserida no espaço peridural era cortada com tesoura estéril, dentro de um tubo seco e enviada para cultura, logo após a retirada.

A metodologia laboratorial utilizada foi recomendada por Maki et al ${ }^{(18)}$ cultura pela técnica semiquantitativa, onde o resultado com crescimento de 15 ou mais unidades formadoras de colônias de determinado organismo deve ser considerado positivo para infecção. Quando a cultura foi positiva e havia presença de um dos sinais flogísticos, foi considerado infecção $0^{(19)}$. Porém, na ausência de sintomas e sinais clínicos de infecção, considerou-se colonização(20). Nos casos em que os pacientes apresentaram febre, foi colhida uma amostra de hemocultura em 2 tubos, com 8 a $10 \mathrm{ml}$ de sangue, para pesquisa de aeróbios e fungos.

\section{RESULTADOS}

Dos 27 pacientes que utilizaram cateter peridural tunelizado, 13 (48,14\%) eram do sexo feminino e 14 (51,85\%) masculino. A média de idade era 61,7 anos, variando de 26 a 82 anos. Em relação ao nível de inserção, 20 (74,04\%) pacientes tinham inserção lombar e 7 (25,9\%) torácica. 0 tempo de permanência do cateter foi em torno de 10,2 dias (variação de 3 a 65 dias -ver detalhes na tabela 1). Não foram utilizados antimicrobianos em nenhum dos casos.

Dentre os 17 pacientes com dor crônica não-oncológica, três eram portadores de polineuropatia diabética, 03 de dor pós-operatória, 03 decorrente de Vasculopatia Isquêmica, 02 eram portadores de dor por apresentarem Distrofia Simpático-Reflexa (Síndrome Complexa de Dor Regional Tipo I), 02 de dor em Fibromialgia, 01 de Síndrome Dolorosa Pós-Laminectomia, 01 de Dor Mielopática secundária a infecção pelo
Tabela 1. Características da amostra estudada. São Paulo, 2004.

\begin{tabular}{lcc}
\hline Características & \multicolumn{2}{c}{ Cateter Tunelizado (n=27) } \\
\cline { 2 - 3 } & {$[\mathrm{n}]$} & {$[\%]$} \\
\hline Sexo & 14 & 51,8 \\
Masculino & 13 & 48,2 \\
Feminino & & \\
\hline Idade & 61,7 & \\
Mediana & 82 & \\
Limite superior & 26 & \\
Limite inferior & & 37 \\
\hline Diagnóstico & 10 & 63 \\
Dor oncológica & 17 & \\
Dor não-oncológica & & 25,9 \\
\hline Local de Inserção & 7 & 74,1 \\
\hline Torácico & 20 & \\
Lombar & & \\
\hline Tempo de permanência (dias) & 10,2 & \\
Mediana & 3 & \\
Limite superior & 65 & \\
Limite inferior &
\end{tabular}

vírus da varicela, 01 de dor decorrente de fratura dorsal e 01 por Mononeuropatia Sural.

Nesse estudo, $04(14,8 \%)$ cateteres foram considerados colonizados e os microorganismos presentes foram Staphylococcus sp em dois pacientes (Pseudomonas aeruginosa e Staphylococcus aureus). Neoplasia em estágio avançado foi a doença primária nos 04 pacientes (tabela 2). Vale ressaltar que não apresentavam sinal de infecção, somente resultado da cultura positiva. Após a retirada do cateter permaneceram sem nenhuma manifestação infecciosa.

Apenas 01 paciente $(3,7 \%)$ apresentou infecção, tendo cultura positiva da ponta do cateter para Staphylococcus sp. A indicação do implante do cateter foi por dor intensa persistente por compressão medular à nível torácico por fratura de vértebra. A paciente estava sem mobilidade devido ao quadro álgico, apresentava início de úlcera por pressão em região sacral e estado geral regular. Após 19 dias de instalação, a paciente apresentou hipertermia $\left(38,5^{\circ} \mathrm{C}\right)$, sendo colhida a hemocultura. Como a febre permanecia, foi retirado o cateter peridural no $23^{\circ}$ dia e enviada a ponta para cultura, a qual apresentou positividade para Staphylococcus sp. O resultado da hemocultura foi negativo e a febre cessou após a retirada do cateter peridural.

\section{DISCUSSÃO}

Os pacientes que utilizaram cateter peridural tunelizado formaram um grupo heterogêneo quanto à patologia, idade, estado geral e tempo de permanência do cateter. A dor foi satisfatoriamente aliviada em todos os pacientes. $O$ paciente que apresentou infecção teve o cateter retirado no $4^{\circ}$ dia de febre, evoluindo sem complicações.

Acreditamos que a baixa taxa de infecção deve-se ao cuidado no momento de instalação do cateter peridural, no Centro Cirúrgico. Porém, ainda devem ser implementados protocolos pelo número de cateteres

Tabela 2. Pacientes colonizados* e infectados*. Sao Paulo, 2004.

\begin{tabular}{|c|c|c|c|c|c|c|}
\hline Paciente & Sexo & Idade & $\begin{array}{l}\text { Tempo de } \\
\text { permanência }\end{array}$ & Nivel de inserção & Patologia & Microrganismo \\
\hline $\begin{array}{l}1 \\
2\end{array}$ & $\begin{array}{l}\mathrm{M}^{\mathrm{x}} \\
\mathrm{F}^{\mathrm{x}}\end{array}$ & $\begin{array}{l}34 \\
72\end{array}$ & $\begin{array}{c}5 \\
19\end{array}$ & $\begin{array}{l}\text { Lombar } \\
\text { Torácico }\end{array}$ & $\begin{array}{l}\text { Sarcoma fêmur } \\
\text { Neo pâncreas }\end{array}$ & $\begin{array}{l}\text { Staphyl.sp } \\
\text { Pseud.aenug }\end{array}$ \\
\hline $\begin{array}{l}3 \\
4 \\
5\end{array}$ & $\begin{array}{l}F^{x} \\
F^{x x} \\
M^{x}\end{array}$ & $\begin{array}{l}87 \\
78 \\
70\end{array}$ & $\begin{array}{c}20 \\
23 \\
3\end{array}$ & $\begin{array}{l}\text { Lombar } \\
\text { Lombar } \\
\text { Lombar }\end{array}$ & $\begin{array}{l}\text { Neo ovário } \\
\text { Fratura vértebra } \\
\text { Neo pulmão }\end{array}$ & $\begin{array}{l}\text { Staphyl.aureus } \\
\text { Staphyl. sp } \\
\text { Staphyl. sp }\end{array}$ \\
\hline
\end{tabular}


colonizados. A orientação continuada à equipe de enfermagem tem sido de grande importância para a manutenção sem intercorrências do cateter por vários dias. 0 cuidado no preparo das soluções analgésicas nas unidades de internação, a troca do equipo a cada 72 h, a observação da pele no local da inserção e a troca do curativo são medidas que fazem do cateter peridural tunelizado um método de analgesia seguro.

Segundo Mann ${ }^{(11)}$, a infusão contínua de analgésicos por via peridural revolucionou a qualidade da analgesia, mas questionava se os mesmos estavam aptos a evitar a infecção peridural. Cita que Yuste et al relataram um caso de abcesso peridural, o qual associaram à disseminação hematogênica que colonizou um local de baixa resistência e que, Darouiche et al também acreditavam ser essa a causa mais freqüente de abcesso peridural. Mann ${ }^{(1)}$ ainda discorda afirmando que. no seu estudo, a infusão contaminada e a manipulação de seringas foram as maiores causas de infecção.

Cabe salientar que, no presente estudo, em um dos casos a apresentar colonização por Staphylococcus sp, o cateter foi utilizado por somente cinco dias, sendo interrompido devido ao óbito do paciente. Em outro caso, 0 cateter permaneceu por 68 dias, sem sinais de contaminação.

Poderia ser considerado que os 04 pacientes que apresentaram colonização são pacientes imunodeprimidos pelo estágio avançado do câncer e provavelmente já se apresentavam colonizados? Byers et al ${ }^{(10)}$ referiram ter baixa taxa de infecção, mesmo considerando a gravidade da doença dos pacientes em tratamento de câncer e com longo tempo

\section{REFERÊNCIAS}

1. Bromage, PR. Analgesia peridural. São Paulo (SP): Editora Manole; 1980

2. White MJ, Lopes KA, Nessly M. Postoperative epidural morphine is safe on surgical wards. Anesthesioloogy 1991; 75: 452-6.

3. Cavalcante VO, Teixeira MJ, Franco RA. Procedimentos anestésicos e o tratamento da dor. In: Teixeira MJ, Figueiró JAB. Dor: epidemiologia fisiopatologia, avaliação, síndromes dolorosas e tratamento. São Paulo (SP): Grupo Editorial Moreira Jr; 1996. p. 160-8.

4. Drummond JP. Dor aguda. Fisiopatologia, clínica e terapêutica. São Paulo (SP): Atheneu; 2000

5. Andrade MP. Dor pós-operatória: conceitos básicos de fisiopatologia e tratamento. Rev Dor 2000; 2(2): 7-14.

6. Teixeira MJ, Braum FHO, Marquez JO, Lin TY, editores. Dor Contexto Interdisciplinar. Curitiba (PR): Editora Maio; 2003.

7. Spiegel $P$, et al. Tratamento da dor com morfina com cateter peridural tunelizado: experiência clínica. Rev Bras Anest 2003 jul-ago; 53 (4): 313-21

8. Chaves LD. Analgesia espinhal e analgesia controlada pelo paciente: aplicacõos e procedimentos para a prática de enfermagem. In: Dor $5^{0}$ sinal vital. Reflexões e intervenções de enfermagem. $1^{\mathrm{a}}$ ed. São Paulo (SP): Editora Maio; 2004. p. 290-303.

9. Fujinaka W, Hinomoto N, Saeki S, Yoshida A, Uemura S. Decreased risk of catheter infection in infants and children using subcutaneous tunneling for continuous caudal anesthesia. Acta Med Okayama 2001; 55(5): 283-7.

10. Byers K, Axelrod P, Michael S, Rosen S. Infections complicating tunneled intraspinal catheter systems used to treat chronic pain. Clin Infec Dis 1995; 21: 403-8.

11. Mann E. Epidural analgesia: have you got right? Nurs Times 1998 aug; 94 (32) 52-4.

12. Grinde JW, Grina R, Gellatly T. Pain management by epidural analgesia: de uso do cateter. Bengtsson ${ }^{(21)}$ concluiu que pacientes com feridas contaminadas têm potencial risco de apresentar infecção no cateter peridural. Em seu artigo, nos 03 casos apresentados de infecção peridural com uso de analgesia pelo cateter, nenhum estava imunodeprimido ou tinha alguma doença associada, como Diabetes Mellitus. Porém, dois pacientes fizeram amputação de membro inferior e o terceiro internou com úlcera infectada no pé.

Smitt et al(22), em estudo com pacientes oncológicos, concluíram que a infecção peridural é freqüente e que, na presença de qualquer sintoma, como dor nas costas persistente, febre ou déficit neurológico deve ser prontamente avaliado por ressonância magnética.

Acreditamos que o acompanhamento rigoroso dos pacientes que estão com cateter peridural tunelizado, os protocolos e o treinamento da enfermagem permitem que a técnica seja benéfica com eficiente controle dos riscos.

\section{CONCLUSÃO}

Observando os cateteres acompanhados, foi evidenciada presença de microorganismos em $\mathrm{N}=5$ (18,51\%), dos quais apenas um paciente $(3,7 \%)$ evoluiu com sinais e sintomas de infecção, resultando na perda do cateter. Comparando os dados encontrados com a literatura, comprovamos que o cateter tunelizado é um método seguro para 0 alívio da dor crônica. Protocolos para o preparo da pele no momento da inserção e para a troca do curativo devem ser implantados, visto que os microorganismos encontrados são colonizantes de pele. the challenge for nursing. Heart Lung 1984 mar; 13(2):105-10.

13. Abreu MP, Deda RG, Cangiani LH, Aquino HMD, Ortiz J. Abscesso peridural após analgesia controlada pelo paciente por via peridural. Relato de caso. Rev Bras Anestesiol 2004; 54(1): 78-83.

14. Burstal R, Wegener F, Hayes C, Lantry G. Epidural analgesia prospective audit of 1062 patients. Anaesth Intens Care 1998 abr; 26(2):165-72.

15. Pimenta AMP, Fukuda CL. Analgesia Peridural Domiciliar: atuação da enfermeira. Rev Bras Enferm 1994 jan-mar; 47(1):31-5

16. Teixeira $\mathrm{MJ}$, et al. Abordagem multi e interdisplinar. In: Dor $-5^{\circ}$ sinal vital. Reflexões e Intervenções de Enfermagem. $1^{a}$ ed. São Paulo (SP): Editora Maio; 2004. p. 33-50.

17. Bernardo CLE. O papel da enfermagem. In: Drummond JP. Dor Aguda: fisiopatologia, clínica e terapêutica. São Paulo (SP): Atheneu; 2000. p. 171-212.

18. Maki DG, Weise CE, Sarafin HW. A semiquantitative culture method for identifying intravenous-catheter-related infecton. N Engl J Med 1977; 296(23): 1305-9.

19. Guidelines for the prevention of intravascular catheter-related infections, do Centers of disease control and prevention. (CDC) 2002 ago 51(RR-10).

20. Ritchmann R, Levin ANS, et al. Infecção relacionada ao uso de cateteres vasculares. Assoc Paul Estudos e Controle Infec Hosp 1999; 1-58.

21. Bengtsson M, Netterblad H, Sjoberg F. Extradural catheter-related infections in patients with infected cutaneous wounds. Britsh J Anaesthesia 1997; 79: 668-70.

22. Smitt PS, Tsafka A, Zande FT. Outcome and complications of epidural analgesia in patients with chronic cancer pain. Cancer 1998 nov; 83(9): 2015-22. 